\title{
Manejo de pacientes anticoagulados y antiagregados: documento de consenso de la SEC y sus implicaciones en Podología
}

\author{
Management of anticoagulated and antiaggregated patients: consensus statement of SEC \\ and implications for Podiatry
}

Cada año aumenta el número de pacientes en tratamiento con fármacos antitrombóticos (anticoagulantes y/o antiagregantes) que atendemos en nuestras consultas. Hasta la fecha, el manejo periprocedimiento de estos pacientes era un problema habitual en la práctica clínica diaria y en nuestro campo no existía un consenso claro sobre cuál debía ser el tratamiento óptimo de estos pacientes en los procedimientos invasivos que realizamos. Recientemente, la Sociedad Española de Cardiología (SEC) ha publicado un documento de consenso que agrupa a expertos de todas las sociedades científicas españolas implicadas en el proceso perioperatorio del paciente con el objetivo de homogeneizar las recomendaciones más importantes sobre cómo debe ser el manejo de los pacientes en tratamiento anticoagulante y anteagregante de un modo sencillo, práctico y fácil de aplicar ${ }^{1}$.

La decisión final de mantener o retirar el tratamiento anticoagulante y antiagregante en estos pacientes de cara a cualquier procedimiento invasivo está determinada, por un lado, por el riesgo trombótico al que se somete al paciente cuando se le retira la medicación y, por otro lado, por el riesgo hemorrágico del procedimiento si la medicación se continúa. De la interacción de estos dos factores depende la actitud a tomar en estos pacientes. El documento cataloga el riesgo de hemorragia de los distintos procedimientos y define como riesgo hemorrágico bajo a todas aquellas intervenciones en las que "la hemostasia se puede conseguir adecuadamente, una posible hemorragia no supone un riesgo vital para el paciente ni compromete el resultado de la cirugía y no requiere transfusión"1. Asimismo, el documento ofrece un listado de procedimientos en todas las especialidades quirúrgicas clasificándolos según su riesgo de hemorragia (bajo, moderado y alto). El documento propone que se valore NO INTERRUMPIR la anticoagulación para procedimientos de bajo riesgo hemorrágico en los que la posible hemorragia derivada del procedimiento es clínicamente poco importante y asumible por el operador. Todos los procedimientos invasivos que realizamos en Podología (infiltraciones anestésicas locales, infiltraciones intralesionales e intrarticulares, biopsias excisionales, injertos y colgajos locales, procedimientos de cirugía ungueal, cirugía osteoarticular de antepié abierta y cerrada por M.I.S. y cirugía osteoarticulary/o reconstructiva de retropié) son catalogados en dicho documento como procedimientos de riesgo hemorrágico bajo, en los que se propone valorar no interrumpir el tratamiento anticoagulante durante el perioperatorio del paciente.

Igualmente, en el manejo periprocedimiento del paciente antiagregado, el documento de consenso recomienda NO INTERRUMPIR la antiagregación cuando el riesgo hemorrágico es bajo en casos de antiagregación simple, especialmente con ácido acetilsalicílico (AAS). En casos de antiagregación plaquetaria doble (generalmente con AAS más inhibidores del P2Y ${ }_{12}$ [clopidogrel, prasugrel o ticagrelor]), la recomendación es posponer el procedimiento electivo hasta que el riesgo trombótico del paciente baje y pueda, por tanto, pasar 
a antiagregación simple. En caso de que el procedimiento no pudiera ser pospuesto, la recomendación de este grupo de trabajo es igualmente NO INTERRUMPIR la doble antiagregación plaquetaria si el riesgo de hemorragia es bajo (todos nuestros procedimientos lo son).

Mi experiencia personal después de tratar pacientes en los que se ha retirado la medicación antitrombótica y pacientes en los que se ha mantenido la medicación antitrombótica durante el periodo perioperatorio es que todos los procedimientos invasivos que realizamos en Podología pueden llevarse a cabo de forma satisfactoria en pacientes anticoagulados y antiagregados (antiagregación simple) sin necesidad de modificar su régimen de tratamiento durante el periodo perioperatorio. La hemorragia derivada del procedimiento es clínicamente poco relevante y asumible en la inmensa mayoría de los casos. Sin embargo, no existen en Podología estudios sobre el manejo periprocedimiento de estos pacientes y animamos a la realización de estudios en este sentido, que aporten evidencias que validen estas recomendaciones avaladas actualmente por 23 sociedades científicas de nuestro país'.

Un saludo,

Javier Pascual Huerta

Redactor Jefe. Revista Española de Podología

\section{BIBLIOGRAFÍA}

1. Vivas D, Roldán I, Ferrandis R, Marín F, Roldán V, Tello-Montoliu A, et al. Manejo perioperatorio y periprocedimiento del tratamiento antitrombótico: documento de consenso de SEC, SEDAR, SEACV, SECTCV, AEC, SECPRE, SEPD, SEGO, SEHH, SETH, SEMERGEN, SEMFYC, SEMG, SEMICYUC, SEMI, SEMES, SEPAR, SENEC, SEO, SEPA, SERVEI, SECOT y AEU. Rev Esp Cardiol. 2018;71(7):553-64. https://www.revespcardiol. org/es-manejo-perioperatorio-periprocedimiento-del-tratamiento-articulo-S0300893218300034 